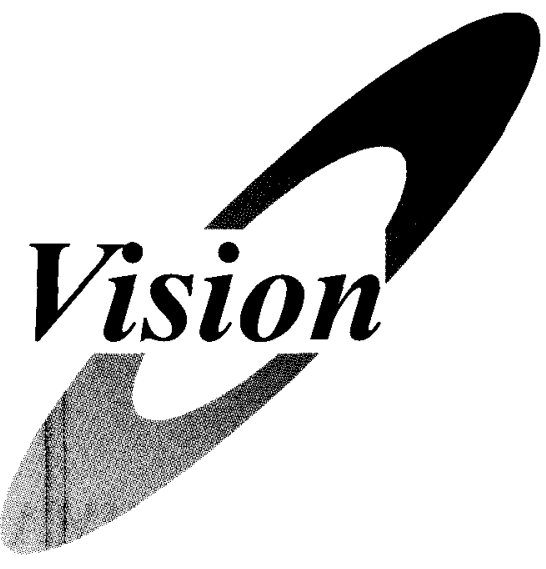

\title{
Harvesting Sunlight by Photoelectrochemical Energy Conversion
}

\author{
Michael GRÄTZEL
}

Ever since Becquerel's discovery of the photoelectric effect in 1839, scientists have been infatuated with the idea of converting light to electric power or chemical fuels. To date the field of photo-energy conversion has been dominated by solid state junction devices, usually made of crystalline or amorphous silicon and profiting from the experience and material availability resulting from the semiconductor industry. This is now being challenged by the emergence of a new generation of cells based for example on mesoporous oxides and conducting polymers films. Their design differs from that of the classical p-n junction in that the contacting phase to the semiconductor is replaced by a liquid, gel or solid electrolyte, thereby forming a photoelectrochemical cell. Contrary to expectations, devices based on interpenetrating networks of mesoscopic semiconductors have shown strikingly high conversion efficiencies, competing with those of conventional silicon. The prototype of this family is the solar cell based on dye sensitized nanocrystalline titanium dioxide films developed in the author's laboratory that reaches efficiencies of 10-11 percent in full sunlight. Present endeavors aim to improve further the efficiency. A large research effort is also under way on solid state cells where the electrolyte is replaced by an inorganic or organic hole conductors.

Despite of this remarkable progress, there is still intensive debate on how photo-energy conversion can occur so efficiently in these systems despite the absence of a local electric field at the site of the junction required in conventional devices to obtain light induced charge separation. Here, electrochemical methodologies, such as intensity modulated photocurrent and photovoltage spectroscopy provide important clues to unravel the fundamental features of the light energy conversion process preparing the ground for the realization of more advanced future generation devices.

This special issue of the journal Electrochemistry on "New Trends in Photo-energy Conversion" pays tribute to the pioneering work that Japanese scientist have carried out in this promising field. Following the discovery of photo induced water cleavage on illuminated $\mathrm{TiO}_{2}$ electrodes by Fujishima and Honda some thirty years ago, the Japans research community has maintained a leading role in the research on light energy conversion, entailing world-wide respect and recognition. The present issue highlights the phenomenal advances made in this important area and outlines future perspectives and opportunities.

Research on photo-energy conversion will intensify in the near future as it is expected to make a pivotal contribution to the sustainable development of human society. The earth's oil reserves are expected to run out during this century. At the same time the energy needs of the planet will at least double within the next 50 years setting the stage for a major supply shortage unless renewable sources can cover the substantial deficit that fossil fuels can no longer furnish. Public concern has heightened due to the disastrous environmental pollution arising from oil spills and the frightening climatic consequences of the green house effect caused by the combustion of fossil fuels. Fortunately the supply of energy from the sun to the earth is about ten thousand times more than what mankind consumes currently. To tap into this huge energy reservoir remains nevertheless a major challenge. Electrochemical research on photoenergy conversion will play a critical role in future efforts to master this important task.

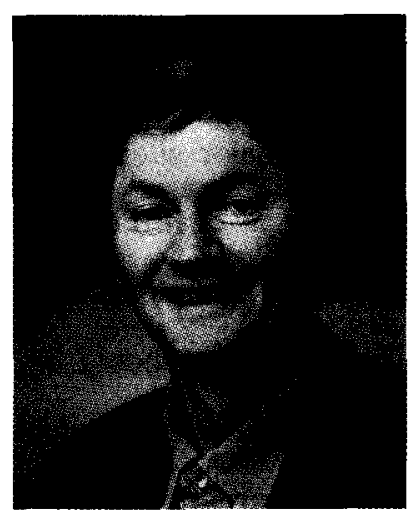

Michael GRÄTZEL Prof. of the Swiss Federal Institute of Technology 